Pacific Journal of Mathematics

METHODS OF SUMMATIO 


\title{
METHODS OF SUMMATION
}

\author{
G. M. PETERSEN
}

1. Methods of Rogosinski and Bernstein. In this note we shall discuss certain matrix methods of summation, though otherwise $\S 1$ and $\S 2$ are unrelated. In this section we wish to consider some of the properties of the method $\left(B^{h}\right)$, where we say that a series $\sum_{\nu=0}^{\infty} u_{\nu}$ is summable $\left(B^{h}\right)$ when

$$
B_{n}^{h}=\sum_{\nu=0}^{n} u_{\nu} \cos \frac{\pi}{2}\left(\frac{\nu}{n+h}\right) \rightarrow S, n \rightarrow \infty
$$

The method $\left(B^{h}\right)$ has been the subject of recent papers by Agnew [1], Karamata $[5,6]$, and Petersen [7]. It has been shown in the papers by Agnew and Petersen that for $h>1 / 2$ the method $\left(B^{h}\right)$ is equivalent to the arithmetic means of Cesaro $(C)$, and in the paper by Agnew that for $0<h<1 / 2$ the method is equivalent to methods stronger than $(C)$.

We shall now construct examples after a method of Hurwitz [4], to show that for $h<0$ the method $\left(B^{h}\right)$ sums a series not summable $(C)$. Hence, since all series summable $(C)$ are summable $\left(B^{h}\right)$, we shall have proved that $\left(B^{h}\right)$ is stronger than $(C)$.

We shall first consider $-1<h<0$, so that all the coefficients in any row are positive except the $n$th coefficient $\cos \{\pi n /[2(n+h)]\}$. We choose $u_{0}>1$ and assume that the first $m-1$ terms of the series $\sum_{\nu=0}^{\infty} u_{\nu}$ are known. Then we select $u_{m}$ so that

$$
B_{m}^{h}=\sum_{\nu=0}^{m} u_{\nu} \cos \frac{\pi}{2}\left(\frac{\nu}{m+h}\right)=0
$$

or

$$
-u_{m} \cos \frac{\pi}{2}\left(\frac{m}{m+h}\right)=\sum_{\nu=0}^{m-1} u_{\nu} \cos \frac{\pi}{2}\left(\frac{\nu}{m+h}\right) .
$$


All of the $u_{\nu}$ are positive; and since

$$
\frac{u_{m}}{u_{m-2}} \geq \frac{\sin \frac{\pi}{2}\left(\frac{2+h}{m+h}\right)}{-\sin \frac{\pi}{2}\left(\frac{h}{m+h}\right)} \simeq-\left(\frac{2}{h}+1\right)
$$

for $-1<h<0$, the $u_{\nu}$ do not satisfy $u_{n}=o(n)$, and hence $\sum_{\nu=0}^{\infty} u_{\nu}$ is not summable $(C)$; see [3].

If $h \leq-1$, we consider

$$
B_{m}^{h}=\sum_{\nu=0}^{m-1}\left[\cos \frac{\pi}{2}\left(\frac{\nu}{m+h}\right)-\cos \frac{\pi}{2}\left(\frac{\nu+1}{m+h}\right)\right] S_{\nu}+\cos \frac{\pi}{2}\left(\frac{m}{m+h}\right) S_{m} .
$$

Here again we select positive increasing $S_{\nu}$ so that $B_{\nu}^{h}=0$ for $\nu \leq m-1$. Under the assumption that $S_{\nu} \geq \nu, \nu \leq m-1$, we shall show that $S_{m} \geq m$. Observing that the first $m-1$ coefficients of the $S_{\nu}$ are positive, we have (setting $\pi /[2(m+h)]=\theta)$ :

$$
\begin{aligned}
-\cos m \theta & \geq \sum_{\nu=0}^{m-1}[\cos \nu \theta-\cos (\nu+1) \theta] \nu \\
& =\sum_{\nu=0}^{m-1} \cos \nu \theta-(m-1) \cos m \theta \\
& =\Re \sum_{\nu=0}^{m-1} e^{i \nu \theta}-(m-1) \cos m \theta \\
& =\Re \frac{1-e^{i m \theta}}{1-e^{i \theta}-(m-1) \cos m \theta}-(m-1) \cos m \theta \\
& =\Re \frac{i\left(e^{-(i \theta) / 2}-e^{i(m-1 / 2) \theta}\right)}{2 \sin \theta / 2} \\
& \geq\left(\frac{1}{2}-\frac{\pi}{2} h\right) ;
\end{aligned}
$$

therefore, 


$$
S_{m} \geq\left(\frac{1}{2}-\frac{\pi}{2} h\right) \frac{m+h}{-h} \times \frac{2}{\pi} \geq q m, q>1 .
$$

Hence the series constructed does not satisfy the condition $S_{n}=o(n)$, and is not summable $(C)$.

2. A Nörlund method. The method defined by

$$
\sigma_{n}=\left(1-\frac{1}{n+3}\right) S_{n}+\frac{1}{n+3} S_{n+1}
$$

has been used as an example in a recent paper by Agnew [2]. We shall treat this method in a manner similar to that in which the method

$$
t_{n}=(1-a) S_{n-1}+a S_{n}
$$

is treated in [7].

THEOREM. If

$$
\sigma_{n}=\left[\left(1-\frac{1}{n+3}\right) S_{n}+\frac{1}{n+3} S_{n+1}\right] \rightarrow \sigma,
$$

then

$$
S_{n}=C \cdot(-1)^{n-1}(n+1) !+\sigma_{n}^{\prime},
$$

where $\sigma_{n}^{\prime}$ is convergent to $\sigma$ and $C$ is a constant.

Proof. Since (we may assume $S_{0}=0$ )

$$
\begin{aligned}
& (n+2) \sigma_{n-1}=(n+1) S_{n-1}+S_{n} \\
& (n+1) \sigma_{n-2}=\quad n S_{n-2}+S_{n-1} \\
& 3 \sigma_{0}=2 S_{0}+S_{1} \text {, }
\end{aligned}
$$

we have 


$$
\begin{aligned}
S_{n}= & (n+2) \sigma_{n-1}-(n+1)^{2} \sigma_{n-2}+n^{2}(n+1) \sigma_{n-3} \\
& \quad-(n-1)^{2} n(n+1) \sigma_{n-4}+\cdots+(-1)^{n-2} 3^{2} \cdot 4 \cdot 5 \cdot 6 \cdots(n+1) \sigma_{0},
\end{aligned}
$$

or

$$
\begin{gathered}
S_{n=(-1)^{n-1}(n+1) !}\left[(-1)^{n-1} \frac{n+2}{(n+1) !} \sigma_{n-1}+(-1)^{n-2} \frac{n+1}{n !} \sigma_{n-2}+\cdots\right. \\
\left.+(-1)^{\nu} \frac{\nu+3}{(\nu+2) !} \sigma_{\nu}+\cdots+\frac{3}{2} \sigma_{0}\right] .
\end{gathered}
$$

Let

$$
(-1)^{\nu} \frac{\nu+3}{(\nu+2) !} \quad \sigma_{\nu}=t_{\nu} \text {; }
$$

since $\sum_{\nu=0}^{\infty} t_{\nu}$ is absolutely convergent $\left(\sigma_{\nu} \rightarrow \sigma\right)$, we may write

$$
\begin{aligned}
& t_{0}+t_{1}+\cdots+t_{n-1}=C-\left(t_{n}+t_{n+1}+\cdots\right) \\
& =C-\frac{1}{(n+1) !}\left[\frac{n+3}{n+2} \frac{(n+2) !}{n+3} t_{n}+\frac{n+4}{(n+2)(n+3)} \frac{(n+3) !}{n+4} t_{n+1}+\cdots\right] \\
& =C-\frac{(-1)^{n}}{(n+1) !}\left[\frac{n+3}{n+2} \sigma_{n}-\frac{n+4}{(n+2)(n+3)} \sigma_{n+1}+\cdots\right]
\end{aligned}
$$

Then

$$
\begin{aligned}
S_{n} & =(-1)^{n-1}(n+1) !\left[t_{0}+t_{1}+\cdots+t_{n-1}\right] \\
& =(-1)^{n-1} \cdot C \cdot(n+1)+\left[\frac{n+3}{n+2} \sigma_{n}-\frac{n+4}{(n+2)(n+3)} \sigma_{n+1}+\cdots\right] \\
& =(-1)^{n-1} \cdot C \cdot(n+1) !+\frac{n+3}{n+2} \sigma_{n} \\
& -\frac{1}{n+2}\left[\frac{n+4}{n+3} \sigma_{n+1}-\frac{n+5}{(n+3)(n+4)} \sigma_{n+2}+\cdots\right] \\
& =(-1)^{n-1} \cdot C \cdot(n+1) !+\frac{n+3}{n+2} \sigma_{n}-\frac{1}{n+2} O(1)
\end{aligned}
$$




$$
=(-1)^{n-1} \cdot C \cdot(n+1) !+\sigma_{n}+o(1) \text {. }
$$

This proves our assertion.

Obvious extensions can be made to the methods

$$
\sigma_{n}=\left[\left(1-\frac{1}{n+k}\right) S_{n}+\frac{1}{n+k} S_{n+1}\right] \text {, }
$$

or to iterations of these methods.

\section{REFERENCES}

1. R. P. Agnew, Rogosinski-Bernstein trigonometric summability methods and modified arithmetic means, Ann. of Math. (2) 56 (1952), 537-59.

2. Equivalence of methods for evaluation of sequences, Proc. Amer. Math. Soc. 3 (1952), 550-56.

3. G. H. Hardy, Divergent series, Oxford, 1949.

4. W. A. Hurwitz, Totally regular methods of summation, Proc. London Math. Soc. (2) 26, (1926), $231-48$.

5. J. Karamata, Sur la sommabilité de S. Bernstein et quelques procédés de sommation qui s'y rattachent, Mat. Sobornik, N.S. 21 (63) (1947), 13-24.

6. J. Karamata, Über die Beziehung zwischen dem Bernsteinschen und Cesàroschen Limitierungsverfahren, Math. Z. 52 (1949), 305.

7. G. M. Petersen, A note on divergent series, Canadian J. Math. 4 (1952), 445-54.

UNIVERSITY OF ARIZONA

UNIVERSITY OF OKLAHOMA 



\title{
PACIFIC JOURNAL OF MATHEMATICS
}

\section{EDITORS}

\author{
M. M. SCHIFFER* \\ Stanf ord University \\ Stanf ord, California \\ E. HewitT \\ University of Washington \\ Seattle 5, Washington
}

\author{
P. P. DILWCR TH \\ California Institute of Technology \\ Pasadena 4, California \\ E. F. BECKENBACH** \\ University of California \\ Los Angeles 24, California
}

\section{ASSOCIATE EDITORS}

$\begin{array}{ll}\text { H. BUSEMANN } & \text { P. R. HALMOS } \\ \text { HERDERT FF.DERER } & \text { HEINZ HOPF } \\ \text { MARSH ALLL IHALL } & \text { R. D. JAMES }\end{array}$

MARSH AL.L II ALL

\author{
R. D. JAMES
}
BORGE JESSEN
PAUL LÉVY
GEORGE PÓLYA

\author{
J. J. STOKER \\ E. G. STRAUS
}
KÖSAKU YOSIDA

\section{SPONSORS}

UNIVERSITY OF BRITISH COLUMBIA CAI IFORNIA ENSTITUTE OF TECHNOLOGY UNIVERSITY OF CALIFORNIA, BERKELEY UNIVERSITY OF CALIFORNIA, DAVIS UNIVERSITY OF CALIFORNIA, LOS ANGELES UNIVERSITY OF CALIFORNI A, SANTA BARBARA UNIVERSITY OF NEVADA OREGON STATE COLLEGE UNIVERSITY OF OREGON
UNIVERSITY OF SOU THERN CALIFORNIA STANFORD RESE.ARCH INSTITUTE STANFORD UNIVERSITY WASHING TON STATE COLLEGE UNIVERSITY OF WASHINGTON

AMERICAN MATHEMATICAL SOCIETY HUGHES AIRCRAFT COMPANY SHELL DEVELOPMENT COMPANY

* To be succeeded in 1955, by H.L. Royden, Stanford University, Stanford, California.

${ }^{* *}$ To be succeeded in 1955, by E.G. Straus, University of California, Los Angeles 24, Calif.

\author{
Vari-Type Composition by \\ Elaine Barth
}

Printed in the United States of America by

Edwards Brothers, Inc., Ann Arbor, Michigan

UNIVERSITY OF CALIFORNIA PRESS * BERKELEY AND LOS ANGELES COPYRIGHT 1954 BY PACIFIC JOURNAL OF MATHEMATICS 


\section{Pacific Journal of Mathematics}

\section{Vol. 4, No. 1 \\ May, 1954}

Hugh D. Brunk, On the growth of functions having poles or zeros on the positive real axis ................................. 1

J. Copping, Application of a theorem of Pólya to the solution of an infinite

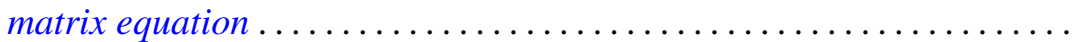

James Richard Jackson, On the existence problem of linear programming ................................... 29

Victor Klee, Invariant extension of linear functionals ............... 37

Shu-Teh Chen Moy, Characterizations of conditional expectation as a transformation on function spaces ..................... 47

Hukukane Nikaidô, On von Neumann's minimax theorem .............. 65

Gordon Marshall Petersen, Methods of summation .................. 73

G. Power, Some perturbed electrostatic fields .................. 79

Murray Harold Protter, The two noncharacteristic problem with data partly

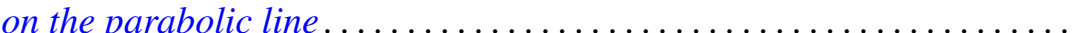

S. E. Rauch, Mapping properties of Cesàro sums of order two of the geometric series........................................... 109

Gerson B. Robison, Invariant integrals over a class of Banach spaces . . . . 123

Richard Steven Varga, Eigenvalues of circulant matrices .............. 151 